\title{
COMPARING THE INFLUENCE OF TWO USER INTERFACES FOR MOBILE RADIOS ON DRIVING PERFORMANCE
}

\author{
Zeljko Medenica and Andrew L. Kun \\ Electrical and Computer Engineering Department \\ University of New Hampshire \\ Durham, NH, USA \\ E-mail: zeljko.medenica@unh.edu, andrew.kun@unh.edu
}

\begin{abstract}
Summary: Mobile radios have a manufacturer-provided manual user interface that allows changing radio channels using buttons. There is also a display on the faceplate of the radio that is used to visually verify channel selection. The objective of this study was to compare the influence of the manual user interface and the Project54 speech user interface (SUI) on drivers' performance while interacting with a mobile radio. In experiments conducted with a driving simulator we found that operating the manual user interface degraded driving performance significantly, while using the SUI did not.
\end{abstract}

\section{INTRODUCTION}

Technological development has introduced a number of electronic devices in police cruisers (lights, siren, radio, video recorder, computer, GPS, etc.). While these devices are important tools in the everyday work of police, they are also potential sources of distraction from the primary task in any vehicle, which is driving. Our hypothesis is that interacting with the devices in the cruiser using a speech user interface (SUI) introduces a much smaller degradation of driving performance than using an interface that requires manual interaction. Note that manual interfaces are often in fact visual/manual interfaces, since they provide visual feedback. Testing our hypothesis involves taking into account many different types of devices and many variables that may influence driving performance (driving task difficulty, recognizer accuracy, etc.). In this paper we describe an experiment to test if our hypothesis holds for user interactions with the police radio under relatively easy driving conditions. Specifically, we compared the influence of a manual user interface and the Project54 SUI on drivers' performance while operating a mobile police radio on a three-lane highway with light traffic. Project54 (Kun et al., 2004) is a software based package that integrates off-the-shelf electronic devices commonly used in police cruisers. The system enables an officer to control these devices using voice commands, while also allowing the officer to use the original manual interfaces provided by the devices' manufacturers.

We chose to examine radio interaction in this study for two reasons. First, the radio is one of the most frequently used devices in the police cruiser. Second, interacting with the radio requires taking one's hand off the wheel and eyes off the road, both of which make crashes more likely. Since police radios have hundreds of channels, these channels are organized into logical groups called zones. Reaching a particular channel requires first selecting the correct zone and then the desired channel. State-of-the-art police radios require officers to use their hands to change zones and channels, which they do by operating hardware buttons on the faceplate of the radio. They also need to look at a display on the faceplate to verify that the correct zone and channel were selected. The Project54 SUI enables officers to issue voice commands for changing zones and 
channels. This means that officers do not have to use the buttons and the display, and can keep their hands on the wheel and eyes on the road.

Many researchers have investigated speech and visual/manual interaction techniques in vehicles and, in general, their conclusions support our hypothesis. Slick et al. (2005) investigated the influence of manual distractions on teenaged drivers' workload. The results showed that subjects maintained the same level of driving performance at a cost of increased workload. Tsimhoni et al. (2004) compared the effects of manual and voice address entry methods on task performance and vehicle control while driving. They used three methods for obtaining input from the user: word-based speech recognition, character-based speech recognition and manual input using the touch-screen keyboard. They found that word-based speech recognition was the fastest input method and it was rated by subjects as relatively safe, followed by character-based speech recognition. On the other hand, manual input was the slowest input method, and it was rated by the subjects as extremely unsafe. Tijerina et al. (1998) performed similar research in order to compare four commercially available route guidance systems. Three systems involved visual/manual methods, while one system involved a voice method of destination entry. Their results showed that the systems with visual/manual destination entry methods had much longer completion times and much more lane departures than the voice system. In some cases speechbased interfaces can introduce a significant cognitive load on a driver, which can undermine driving safety. Lee et al. (2001) performed a study that evaluated the influence of simple and complex speech-based e-mail systems on drivers' reactions to a periodically braking lead vehicle. Their conclusion was that special attention should be paid to the design of a speech user interface in order to avoid unnecessary driver distractions.

\section{METHOD}

In order to evaluate the effects of user interface type on driving performance, we conducted a repeated measures experiment in which subjects performed manual and speech interactions in separate experiments. All subjects performed the manual interactions first, and the time between the experiments was longer than one month. The independent variable was the type of the interaction. The dependent variables, which were used for assessing driving performance, consisted of velocity, car lane position, and steering wheel angle. The experiment was conducted using a high-fidelity driving simulator with a $180^{\circ}$ field of view and a motion base, shown in Figure 1. For the purpose of the experiment, a three-lane straight highway was created. In order to increase the fidelity of the simulation, light ambient traffic was introduced.

The experiment was based on the primary/secondary task paradigm. The primary task was driving while following a lead vehicle at a constant speed of $89 \mathrm{~km} / \mathrm{h}(55 \mathrm{mph})$ and maintaining a constant distance behind it. During the experiment, the participants did not receive any reminders about their performance on the primary (driving) task. The secondary task consisted of changing channels and zones on a mobile police radio. 


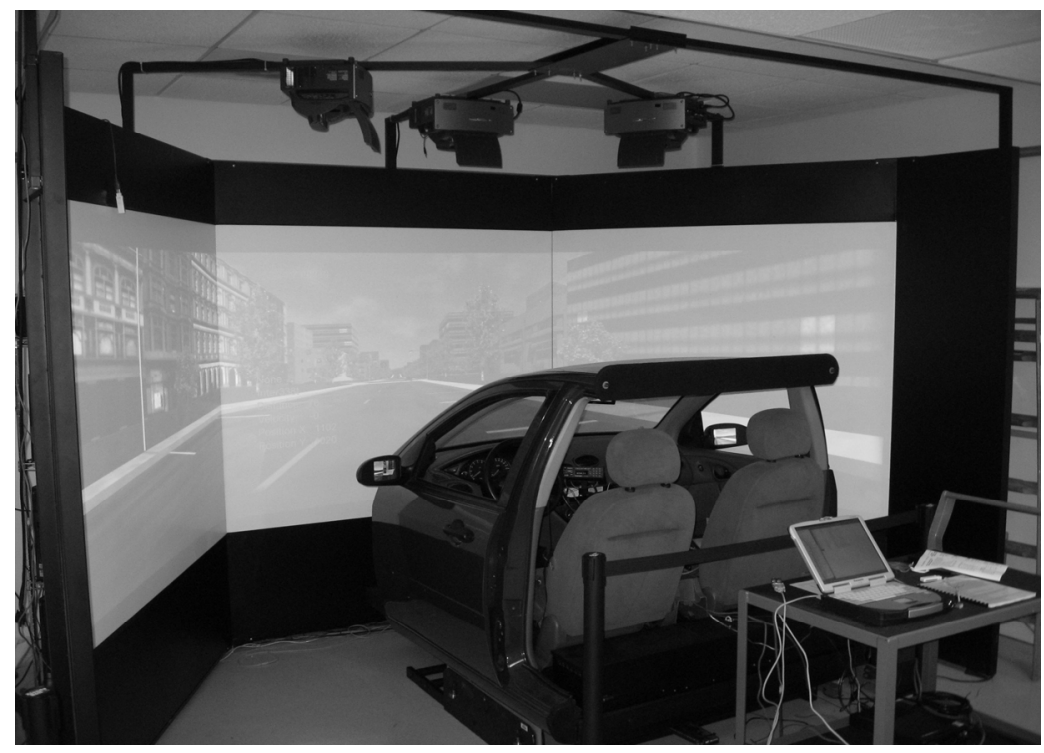

Figure 1. Driving simulator

The secondary task was performed both using the hardware controls installed on the radio faceplate (manual interaction), and using the Project54 SUI (speech interaction). The experimental setup is depicted in Figure 2. In the case of manual interaction, subjects used the buttons (zone up/down and channel up/down) and the display on the radio control head. In the case of speech interaction, subjects issued two types of commands to the SUI, one designating the desired zone and the other the desired channel within that zone. The Project54 SUI also has a push-to-talk button on the steering wheel that had to be pressed while issuing a command. The uttered commands were picked up by the directional microphone which was mounted behind the visor above the driver's head.
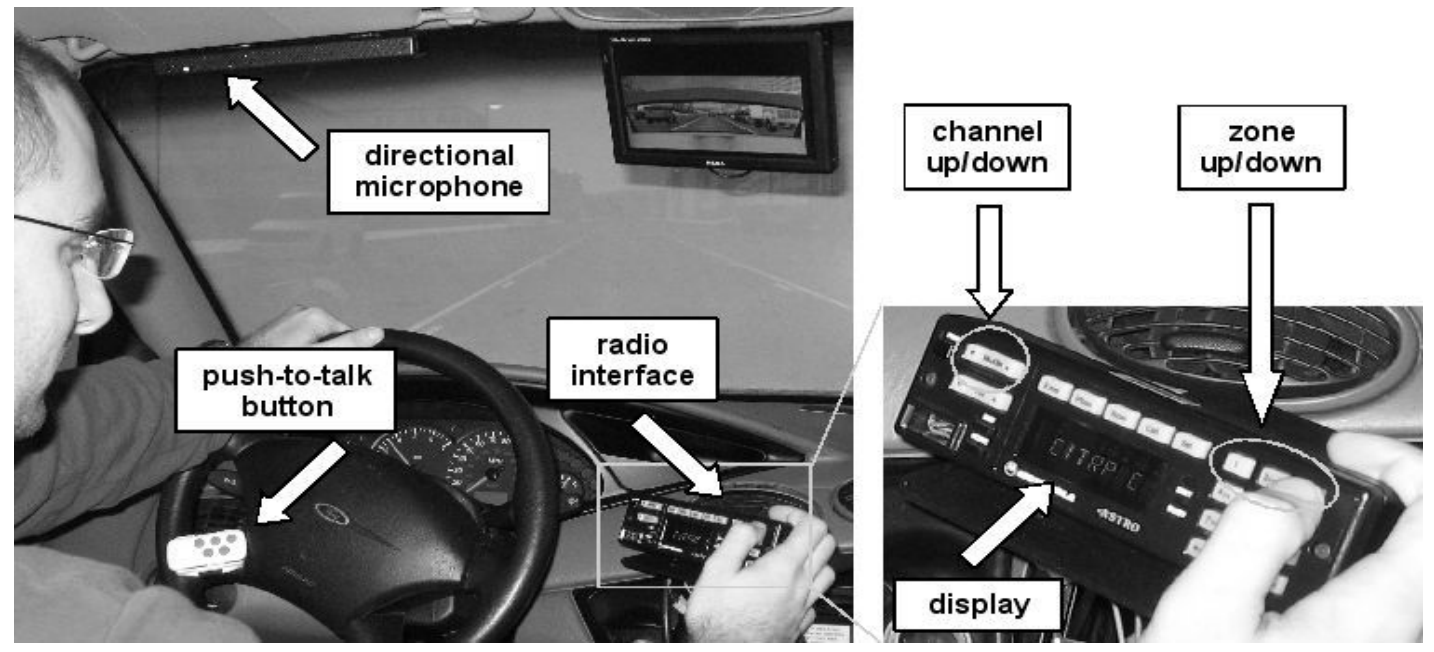

Figure 2. Subject manually adjusting the channels on the radio in the simulator

Eight male university students, between 20 and 27 years of age (their mean age was 23.6 years), all with valid driver's licenses, were recruited to participate in the experiment. All of the participants had normal or corrected to normal vision. Before each experiment, every subject was 
given a training session in order to become accustomed to the simulator and the controls used for operating the radio. Experiments started with the subjects performing the driving task for two minutes, in what we called the baseline condition. Experiments continued with the task condition when the subjects interacted with the radio. Each subject changed channels ten times. Since changing the channel involves changing the zone and then finding the appropriate channel, each subject found ten zones and ten channels for each interaction modality. Clearly, police officers need training in order to be able to quickly navigate between zones and channels. In our experiment, this training was not necessary. Instead, the experimenter prompted subjects to change zones and channels by providing the zone and channel names.

Sample interactions for the manual and the SUI interaction are shown in Figure 3 and Figure 4, respectively. In both figures " $E$ " denotes an utterance by the experimenter, " $S$ " denotes either a visual/manual action or an utterance by the subject and " $C$ " denotes an utterance by the computer. In the case of the manual interaction (Figure 3), in line 1 the experimenter instructs the subject to find the Troop A zone. Line 2 shows that the subject does this by using the radio buttons and display. The experimenter received feedback from the radio via a computer program and could confirm visually when the subject accomplished this task. Once this was the case, the experimenter prompted the subject to find the Rockingham $\mathrm{C}$ channel (line 3). Again, this was done by the subject using the radio buttons and display (line 4). In the case of the sample speech interaction (Figure 4), in line 1 the experimenter tells the subject the speech command to be issued to the SUI in order to reach the Troop B zone. Line 2 shows the subject issuing this command and line 3 shows the SUI echoing the correctly recognized command. In line 4 , the experimenter tells the subject the speech command to get to the Londonderry channel, which the subject issues and the SUI echoes (lines 5 and 6 respectively). The subject has to operate the press-to-talk button when issuing commands. This is denoted with a * in lines 2 and 5.

1. E: Switch Troop A Adam

2. S: Click zone up/down buttons and look at display on radio

3. E: Channel Rockingham C

4. S: Click channel up/down buttons and look at display on radio

Figure 3. Sample manual interaction
1. E: Switch Troop B Boston
2. S: Switch Troop B Boston *
3. C: Switch Troop B Boston
4. E: Channel Londonderry
5. S: Londonderry *
6. C: Londonderry

Figure 4. Sample speech interaction

Immediately after finishing the experiment, each subject filled out the NASA-TLX questionnaire (www.nrl.navy.mil/aic/ide/NASATLX.php) in order to subjectively assess the workload they experienced during the experiment. The questionnaire consists of six scales (mental demand, physical demand, temporal demand, performance, effort, and frustration), and on each scale one can select among 20 different discrete values.

\section{RESULTS}

The dependent variables (velocity, car lane position, and the steering wheel angle) were sampled at a frequency of $10 \mathrm{~Hz}$. The driving performance estimation was based on calculating the variability of the collected data. The less the data varies, the better the performance. Variances were calculated for all three conditions (baseline, manual interaction, and speech interaction), 
and they were analyzed by means of the analyses of variance using the JMP 6.0 statistical software by the SAS Institute. We found no statistically significant difference between variances for data collected under the baseline conditions and during speech interactions. However, there was a highly significant effect of the task condition (manual vs. SUI) on the variability of all collected variables: velocity $(\mathrm{p}<0.00035)$, car lane position $(\mathrm{p}<0.0001)$, and steering wheel angle $(\mathrm{p}<0.000031)$. The mean variances among all subjects for the car lane position, velocity, and steering wheel angle for both task conditions (manual and SUI) are depicted in Figure 5, Figure 6, and Figure 7, respectively.

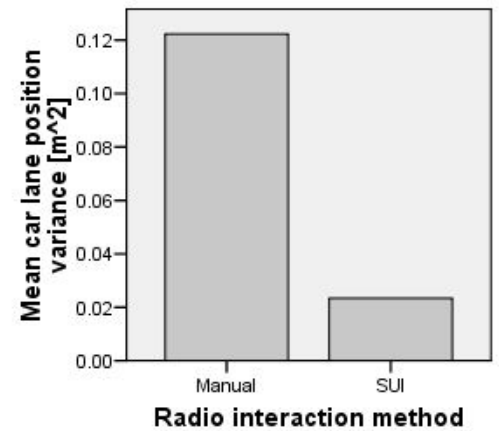

Figure 5. Mean car lane position variance

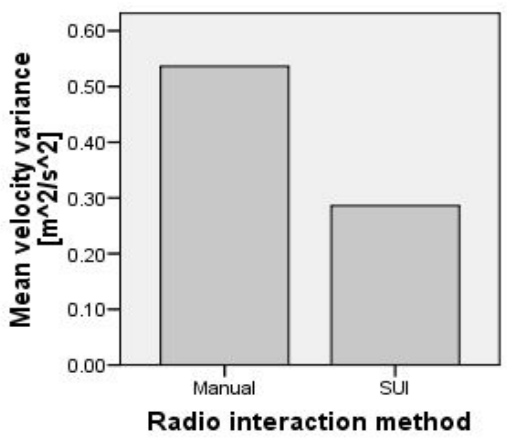

Figure 6. Mean velocity variance

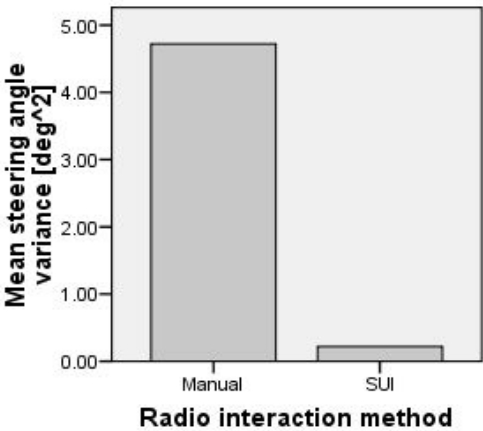

Figure 7. Mean steering wheel angle variance

A reasonable explanation for the above results is that manual interaction with the police radio required releasing the steering wheel and at the same time looking away from the road (as can be clearly seen in Figure 2), and this had a detrimental effect on driving performance. For most subjects, changing channels manually resulted in drastic changes in driving performance between the baseline and the manual interaction task condition that could be observed even by just plotting the time graphs for the dependent variables. The car lane position, recorded from one of the subjects, during the manual interaction experiment is depicted in Figure 8.

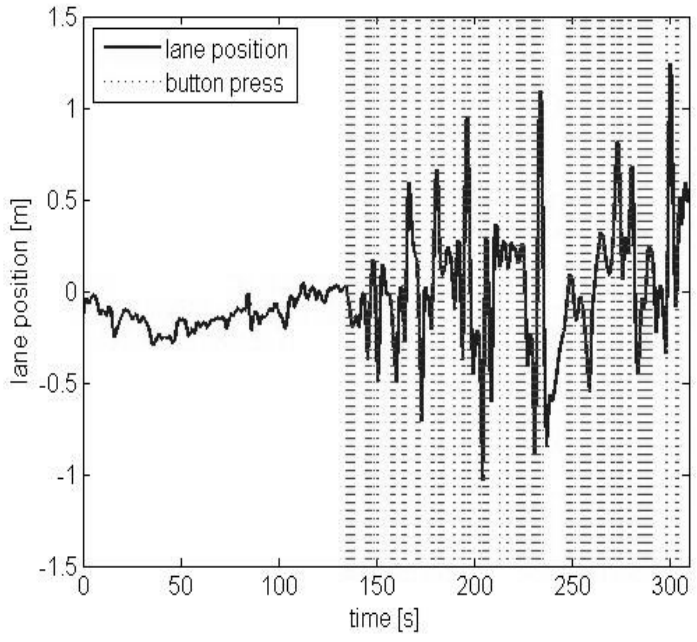

Figure 8. Lane position of a typical subject during manual interaction

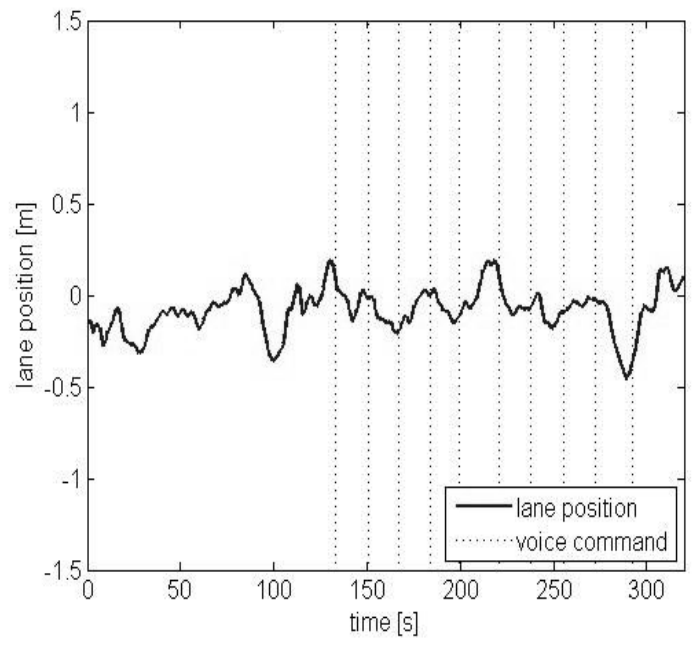

Figure 9. Lane position of the same subject during SUI interaction 
The vertical dotted lines in Figure 8 represent the instants in time when the subject pressed a button on the radio control head. (Note that there were many consecutive button presses for each zone/channel change and thus there are many vertical lines very close together.) At the beginning of the experiment we see the two-minute baseline when the subject was driving without performing any interactions. As we can see in Figure 8, there is a drastic change in the car lane position when the subject started the manual interaction with the radio. The lane position of the same subject, while performing the SUI interaction, is depicted in Figure 9. Here, dotted lines represent the beginning of a speech interaction of the type shown in Figure 4. As we can see in Figure 9, there is basically no change in the car lane position between the baseline and the SUI interaction conditions. Even without performing any calculations, by comparing the two car lane positions in Figure 8 and Figure 9, we can say that the SUI interaction did not introduce any additional variation of the lane position, while the manual interaction did.

By filling out the NASA-TLX questionnaire, all participants reported that they experienced a much higher workload during manual interaction. The mean NASA-TLX workload ratings among all subjects are depicted in Figure 10.

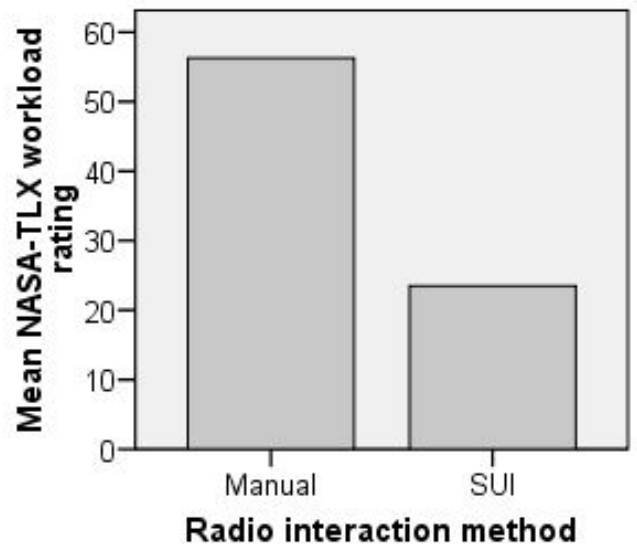

Figure 10. Mean NASA-TLX workload ratings

\section{CONCLUSIONS}

In this paper we present an experimental comparison of the influence of using a manual user interface and using the Project54 SUI on drivers' performance while operating a mobile police radio. Our results show that the interaction type has a significant influence on driving performance. Manual interaction introduced a significant degradation of driving performance, while using the SUI did not. For police mobile radios, the implication is that speech user interfaces are likely to degrade driving performance significantly less than the manual interfaces that are currently the standard on these devices. The general implication for devices designed for the automotive environment is that even manual interfaces that the driver can reach easily (see Figure 2) may degrade driving performance significantly, especially if the driver needs to receive visual feedback from the interface. 


\section{FUTURE WORK}

Our experiment was performed on a straight road with light ambient traffic. An important question is how the observed results would change in more difficult driving conditions. We hypothesize that in more difficult driving conditions the visual/manual interaction would again degrade driving performance more than speech interaction. Another interesting question is how much of the driving performance degradation was due to needing the visual feedback from the radio's visual/manual interface. We are currently looking at repeating the experiment using different in-car mobile devices with manual interfaces that can be operated without the need for visual feedback. One example of such a device is the manual interface for the police lights and siren. With some training, the buttons and levers of such an interface can be operated based on tactile feedback alone. Another important aspect of speech user interfaces that can have an influence on driving is the recognition accuracy of the speech recognizer. In our experiment speech recognition accuracy was $100 \%$. However, speech interaction is a relatively new concept that still has not achieved the level of accuracy that can be absolutely reliable in all situations. There are many factors that can cause speech recognition to fail, especially in vehicles. In these situations a driver has to recover from the error by, for example, repeating a command. We hypothesize that these recognition errors, and the subsequent dialog repairs, can have a negative influence on driving performance. This is the subject of our current research.

\section{ACKNOWLEDGMENTS}

This work was supported by the US Department of Justice under grants 2005CKWX0426 and 2006DDBXK099. The authors would like to thank Professor Philip J. Ramsey for useful suggestions about the statistical analysis.

\section{REFERENCES}

Kun, A.L., Miller, W.T. III, and Lenharth, W.H. (2004). Computers in Police Cruisers. IEEE Pervasive Computing, 3(4), $34-41$.

Lee, J.D., Caven, B., Haake, S., Brown, T.L. (2001). Speech-based Interaction with In-vehicle Computers: The Effect of Speech-based E-mail on Drivers' Attention to the Roadway. Human Factors, 43, 631-640.

Slick, R.F., Cady, E.T., Tran, T.Q. (2005). Workload Changes in Teenaged Drivers Driving with Distractions. Proceedings of the Third International Driving Symposium on Human Factors in Driver Assessment, Training and Vehicle Design, Rockport, Maine, 158-164.

Tsimhoni, O., Smith, D., and Green, P. (2004). Address Entry while Driving: Speech Recognition versus a Touch-screen Keyboard. Human Factors, 46(6), 600-610.

Tijerina, L., Parmer, E., Goodman, M. (October, 1998). Driver Workload Assessment of Route Guidance System Destination Entry while Driving: A Test Track Study. Proceedings of the 5th World Congress on Intelligent Transport Systems, Seoul, Korea 